\title{
PREPARATION AND CHARACTERIZATION OF CELLULOSE NANOFIBERS ISOLATED FROM LETTUCE PEEL
}

\author{
GAONAN ZHANG, ${ }^{* \#}$ FENGRUI WU, ${ }^{* \#}$ TINGTING MA, BINJIA ZHANG, \\ ANNE MANYANDE ${ }^{* *}$ and HONGYING DU, ${ }^{*, * * *}$ \\ *College of Food Science and Technology, Huazhong Agricultural University, \\ Wuhan, Hubei Province 430070, P.R. China \\ ${ }^{* *}$ School of Human and Social Sciences, University of West London, Middlesex, TW89GA, UK \\ ${ }^{* * *}$ Key Laboratory of Environment Correlative Dietology, Ministry of Education, \\ Huazhong Agricultural University, Wuhan, Hubei 430070, P.R. China \\ ${ }^{\#}$ Authors contributed equally to the work \\ $\bowtie$ Corresponding author: Hongying Du, hydu@mail.hzau.edu.cn
}

Received January 18, 2018

Cellulose nanofibers (CNFs) were isolated from lettuce peel using specific chemical treatments, i.e., NaOH alkali solution treatment, $\mathrm{NaClO}_{2}$ bleaching and sulfuric acid hydrolysis. Both cellulose and cellulose nanofibers were characterized by Fourier transform infrared spectroscopy (FTIR) and X-ray diffraction (XRD). The morphological features and particle size distribution of cellulose and nanostructures were explored using transmission electron microscopy (TEM), scanning electron microscopy (SEM) and a Malvern laser particle size analyzer. The results showed that the particle diameters of the majority of nanofibrillated celluloses were in the range from 10 to $20 \mathrm{~nm}$, and the length between 190 and $460 \mathrm{~nm}$. The crystallinity index reached $41.57 \%$, which increased by $117.3 \%$, compared with the raw material. The crystalline lettuce peel nanofibers belong to cellulose type I. Moreover, the thermal degradation temperature of CNFs is $271.7{ }^{\circ} \mathrm{C}$, which is $47.3{ }^{\circ} \mathrm{C}$ higher than that of the untreated peel. The CNFs obtained from these treatments can be used as additional reinforcement in biocomposites in composite manufacturing processes or in the food industry.

Keywords: lettuce peel, cellulose nanofibers (CNFs), isolation, chemical treatment

\section{INTRODUCTION}

Cellulose is the most abundant natural biopolymer, consisting of highly stereo-regular and hydrogen bonded $\beta(1,4)$-glycosidic linked Dglucose chains, organized in hierarchical structures of nanofibrils (3-4 nm), microfibers (10-30 nm) and microfibers (over hundreds of $\mathrm{nm}$ and microns). ${ }^{1-3}$ Currently, cellulose nanofibers (CNFs) have become one of the most attractive renewable resources due to their extraordinary optimal, thermal and mechanical properties, therefore they are regarded as potential biological enhancing nanomaterials by many scientists. ${ }^{4}$ They can be isolated from some green plants by overcoming the extensive and strong inter-fibrillar hydrogen bonds with harsh caustic chemicals, specific enzymes and intense mechanical forces. ${ }^{5}$

In recent years, more and more researchers have focused on the study of cellulose nanofibers, compared with any other chemical materials. The major reason is that CNFs have many advantages that cannot be ignored, namely, they are renewable, strong, have low density and low cost, etc. ${ }^{6}$ These new nanomaterials could be widely used in some fields, including the structuring of plastic, intelligent coatings, cosmetics, pharmaceutical and solar energy collection and so on. ${ }^{7-9} \mathrm{Up}$ to now, there are many by-products of the agricultural industry successfully used to extract CNFs, such as cotton, ${ }^{10,11}$ sugarcane bagasse, ${ }^{12}$ bamboo, ${ }^{13}$ rice straw, ${ }^{2}$ rice husk, ${ }^{14}$ pineapple leaf, ${ }^{15}$ grape skins, ${ }^{16}$ tomato peels,${ }^{9}$ potato peel, ${ }^{17}$ banana peel, ${ }^{18}$ cassava, ${ }^{19}$ rose,${ }^{20}$ etc . However, to our knowledge, there has been no report about cellulose nanofibers isolated from lettuce peel so far.

Lettuce (Lactuca sativa) is one of the favorite vegetables in China, it is widely used both as a vegetable and in traditional medicine. ${ }^{21,22}$ Most 
Chinese people think that lettuce can improve the digestive system and liver function; also, it is especially beneficial to patients with high blood pressure and heart diseases due to its high content of potassium. The edible part of lettuce is mainly in the succulent stems and lettuce leaves, which can be eaten fresh/raw, in salads, fried, dried or pickled. ${ }^{23}$ The waste peel has been applied in sewage treatment as an sorbent, ${ }^{24}$ since it has potential feasibility of a low cost biomaterial. Furthermore, lettuce peel, which accounts for $30 \%-40 \%$ of the raw material, is discarded as waste in most countries. The main aim of this work has been to develop a new application of lettuce peel with high added value, thus avoiding resource waste and environmental pollution. Moreover, lettuce peel contains a high content of cellulose $(54.58 \%)$, compared with other food byproducts. ${ }^{25}$ Furthermore, the extraction of cellulose from lettuce peel is easy to process since common steps are used to obtain CNFs. Using a combination of optimum extraction conditions, the soluble dietary fiber (SDF) ratio could reach $6.96 \%$, and the yield of insoluble dietary fiber (IDF) around $47.62 \% .^{25}$ The expectation is to find a rational and sustainable utilization of lettuce peel with high added value, to meet the needs of the modern food industry.

In this study, the aim has been to isolate CNFs from lettuce peel using several steps, such as alkaline hydrolysis, acid hydrolysis and sodium chlorite bleaching process, to make sure that all the hemicellulose and lignin can be effectively removed. ${ }^{17,26}$ Then, cellulose nanofibers will be obtained following well-established sulfuric acid hydrolysis procedures, which have been applied in the preparation of CNFs from coconut husk, as reported by Rosa. ${ }^{27}$ The morphology and physicochemical properties of cellulose nanofibers are characterized by transmission electron microscopy (TEM), zeta potential nanoparticle size analyzer, Fourier transform infrared spectroscopy (FTIR), X-ray diffraction (XRD) and thermal gravimetric analysis (TGA) techniques. The present method provides a new biopolymer from common vegetable waste, and is expected to be applied in food production as a new natural food additive.

\section{EXPERIMENTAL}

\section{Materials}

Lettuce peel was collected form canteens located by Huazhong Agricultural University, P. R. China. All the water used was purified by an SROP water purification system.

Sodium chlorite $\left(\mathrm{NaClO}_{2}\right)$ and sulfuric acid $\left(\mathrm{H}_{2} \mathrm{SO}_{4}\right)$ were purchased from Shanghai McLean Biochemical Technology Co., Ltd. Sodium hydroxide, glacial acetic acid and ethanol were all of analytical grade and purchased from Chinese Medicine Group Chemical Reagent Co., Ltd. Regenerated cellulose tubing (MW $12,000-14,000$ Da) was obtained from Biosharp Company.

\section{Bran preparation}

Fresh lettuce peels were arranged on aluminum trays and dried in an oven (DHG-9240A, Instrument factory, Shanghai), with forced convection at $45^{\circ} \mathrm{C}$ for $12 \mathrm{~h}$ until the basic water evaporated. The dried peels were ground with a knife mill (81M/AF-10, Western Instrument Technology Co., Ltd), then the powder was passed through a 40-mesh sieve, packed in polyethylene bags and stored at $4{ }^{\circ} \mathrm{C}$ for further analysis.

\section{Isolation of cellulose fibers from lettuce peel}

In order to eliminate the non-cellulosic components, the bran of lettuce peel underwent several steps of chemical treatments under optimized parameters, according to former publications. ${ }^{28,29}$ Firstly, it was processed by alkali treatment with $0.5 \mathrm{~mol} / \mathrm{L} \mathrm{NaOH}$ solution $(w / v$ ratio of $1: 50)$ at $80{ }^{\circ} \mathrm{C}$, and the suspension was mechanically stirred for $2.5 \mathrm{~h}$. The slurry was filtered and washed with distilled water until it reached a neutral $\mathrm{pH}$. This procedure was carried out three times. Secondly, the wet pulp fibers were diluted with $2.3 \mathrm{wt} \% \mathrm{NaClO}_{2}$ solution at $75{ }^{\circ} \mathrm{C}$, and the suspension was mechanically stirred for $3 \mathrm{~h}$, the insoluble material was collected and thoroughly washed with deionized water until neutrality was reached. Then, this step was repeated three times until the color of the pulp totally turned to white. Finally, the purified cellulose was lyophilized (Avanti J-26XP, Beckman, USA).

\section{CNF preparation}

The cellulose was hydrolyzed using $50 \mathrm{wt} \% \mathrm{H}_{2} \mathrm{SO}_{4}$ at a $10 \mathrm{~mL} / \mathrm{g}$ acid-to-cellulose ratio at $50{ }^{\circ} \mathrm{C}$, stirring for $1.5 \mathrm{~h}$. The acid hydrolysis treatment was ceased by adding 10-fold deionized water. The diluted suspension was centrifuged at $9000 \mathrm{rpm}$ to separate the sediments and obtain ivory-colored CNFs. The CNF aqueous suspension was dialyzed against distilled water, using regenerated cellulose tubing for several times until it reached a neutral $\mathrm{pH}$. Finally, the samples were dried in the lyophilizer to obtain the CNFs, and the products were stored under vacuum for further analysis. For simplicity and clarity, the whole procedure for preparing CNFs is presented in Figure 1.

\section{Characterization of morphological features}

The microstructure and morphology of the cellulose microfibers were characterized by a scanning 
electron microscope (SU8010, Hitachi, Japan) at $10 \mathrm{kV}$ accelerating voltage. Samples were fixed on a metal stub with conductive carbon tape and covered with a gold layer.

The transmission electron microscopy (TEM) technique could furnish effective information for the dimension measurement of CNFs. For the analyses, every suspension was placed under ultrasound for 5 min. Then, a drop $(8 \mu \mathrm{L})$ of $0.01 \mathrm{wt} \%$ aqueous suspension was deposited on a 100-mesh copper grid and allowed to dry. The morphology of the CNFs was evaluated using a transmission electron microscope (H-7650, Hitachi, Japan), at an accelerating voltage of $100 \mathrm{kV}$. The TEM images were observed at a scale bar, $200 \mathrm{~nm}$ and the image resolution was set to $1024 \times$ 1024 pixels.

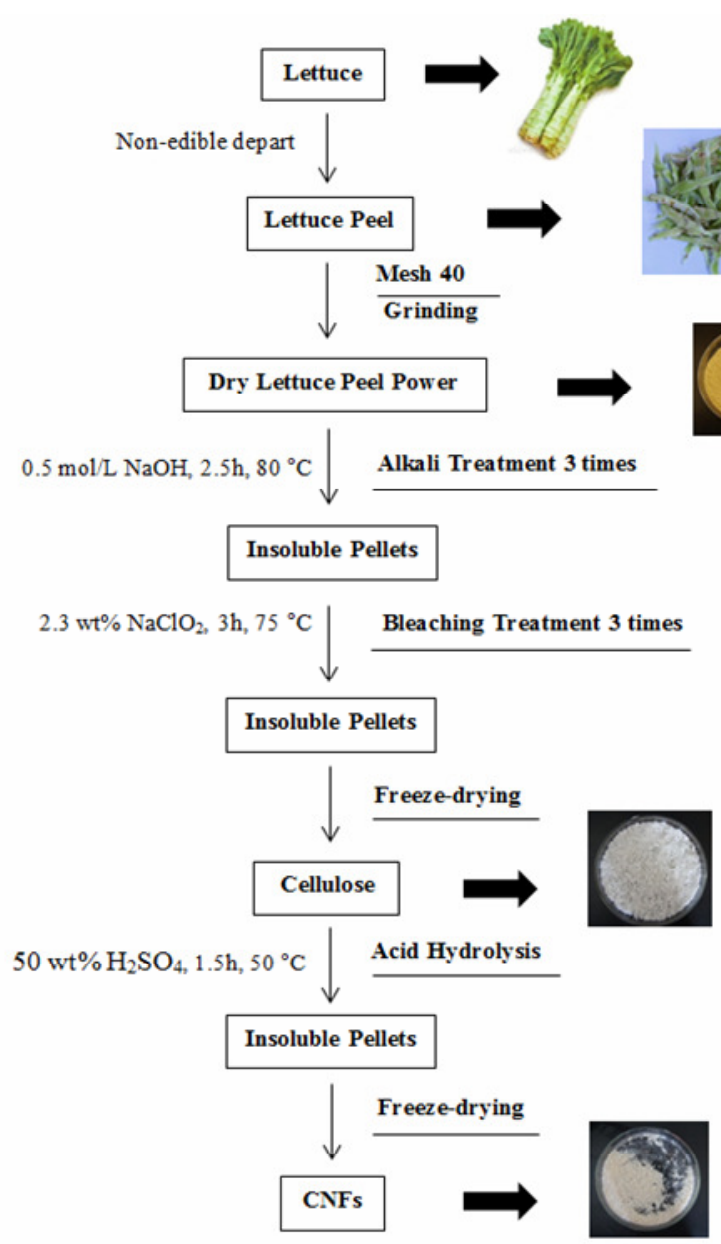

Figure 1: Detailed flow chart of isolation of CNFs generated from lettuce peels and photographic images of corresponding materials in each step

\section{Particle size analysis}

A $0.1 \%(w / v)$ suspension of the sample was used to determine the particle size. The particle size distribution was measured by the dynamic light scattering (DLS), using a Zetasizer (ZEN3600, Malvern, U.K.), with a detection range of $0.6 \mathrm{~nm}-6$ $\mu \mathrm{m}$.

\section{Fourier transform infrared spectroscopy (FTIR)} analysis

FTIR analysis of CNFs at each stage of treatment was carried out to examine the alterations in the structure of the fibers before and after the treatments. FTIR spectra were recorded using an FTIR spectrometer (SX-170, Nicolet, USA) under proper conditions. Samples were dried, ground into a fine powder using a mortar and pestle, mixed with $\mathrm{KBr}$ $(1: 100, w / w)$ and pressed into thin pellets. FTIR spectra were collected in the transmittance mode from an accumulation of 64 scans and recorded in the range of $400-4000 \mathrm{~cm}^{-1}$ at a $4 \mathrm{~cm}^{-1}$ resolution. 


\section{X-ray diffraction (XRD) analysis}

The crystallinity of the samples was investigated by X-ray diffraction (XRD). The crystallinity index (Icr \%) of the lettuce peel bran, cellulose and CNFs was determined by an X-ray diffractometer (D8ADVANCE, Bruker, Germany), using Ni-filtered $\mathrm{Cu}$ $\mathrm{K} \alpha$ radiation $(\lambda=0.15418 \mathrm{~nm})$ at the anode voltage and current of $40 \mathrm{kV}$ and $40 \mathrm{~mA}$, respectively. All the samples were measured in the $2 \theta$ scale of $5^{\circ}-40^{\circ}$ with a scan rate of $2 \% \mathrm{~min}$. The crystallinity index of each sample was calculated by using the following empirical equation (1): ${ }^{30}$

$I_{C F}=\frac{I_{200}-I_{\text {non-er }}}{I_{\text {200 }}} \times 100$

where $I_{200}$ is the diffraction intensity close to $2 \theta=22^{\circ}$ $24^{\circ}$, which represents a crystalline material; and $I_{\text {non-cr }}$ is the diffraction intensity close to $2 \theta=18^{\circ}$, which refers to the amorphous material in cellulosic fibers.

\section{Thermogravimetric analysis}

The degradation characteristics (or thermal stability) of cellulose fibers obtained from different chemical treatments were investigated by thermogravimetric analysis (TGA), using a thermogravimetric analyzer (STA449F3, Netzsch, Germany), with a weight of around $5 \mathrm{mg}$ in a clean platinum pan. The samples were heated from $40{ }^{\circ} \mathrm{C}$ to $700{ }^{\circ} \mathrm{C}$, at a heating rate of $10^{\circ} \mathrm{C} / \mathrm{min}$ under nitrogen atmosphere.

\section{Statistical analysis}

All the experiments were carried out in triplicate. The data of chemical composition were expressed as mean \pm standard deviation. The results were analyzed by analysis of variance (ANOVA). Duncan's multiple comparison tests were used to determine the significant difference between the mean values. A confidence level of $95 \%(\mathrm{p}<0.05)$ was used and the analyses were carried out using SPSS 17.0 software (SPSS Inc., Chicago, USA).

\section{RESULTS AND DISCUSSION Morphological analysis}

The SEM and TEM micrographs of lettuce peel fiber morphological characteristics taken before and after chemical treatment, including bran, cellulose and CNFs, are displayed in Figure 2. It can be seen that the morphological features of these different samples are quite different. The surface of the lettuce peel bran looks smooth and the slender fibrils are arranged together, as shown, in tens of the micrometer scale in Figure 2A. The micrographs of cellulose isolated from the lettuce peel are shown in Figure 2B, it appears as though many cellulose microfibrils are arranged in parallel, but overall the surface of the cellulose looks rough. This phenomenon indicates that the non-cellulosic components, such as pectin, hemicellulose, lignin, wax and other impurities, are partially dissolved and the swelling of cellulose takes place after alkali treatment. ${ }^{31}$

The TEM image of cellulose nanofibers subjected to acid hydrolysis from the lettuce peel cellulose is shown in Figure 2C. It can be seen that through chemical treatment, all the amorphous compounds (lignin, pectin, hemicellulose) have been removed, and only needle-like cellulose nanofibers were left. ${ }^{32}$ The diameter of cellulose fibers is demonstrated in Figure 2C, most of them exhibit a relatively uniform size, and the length and width ranged from 190-460 $\mathrm{nm}$ and 10-20 nm, respectively. $\mathrm{Chen}^{33}$ also reported similar findings on cellulose nanofibers obtained from wood. Under the effect of hydrolysis by sulfuric acid, hydrogen ions enter the non-crystalline region, and destroy the hydrogen bonds and glycosidic bonds, while the crystalline region of cellulose resisted and achieved a nanometer level. In addition, the morphology features of CNFs extracted from lettuce peel were similar but wider $(10-20 \mathrm{~nm})$ than the cellulose nanowhiskers $(\sim 5 \mathrm{~nm})$, which were derived from coconut husk fibers. ${ }^{27}$

\section{Size distribution}

In general, the particle size distribution by the number of nanoparticles can be reflected through dynamic light scattering (DLS), using a Zetasizer. The particle size distribution for the CNFs (length) isolated from lettuce peel after the chemical treatments is illustrated in Figure 3. It had unimodal distribution, which means the particle size of CNFs was relatively uniform. The length of CNFs was distributed in the range of 190-460 $\mathrm{nm}$ and the average value was $280.3 \mathrm{~nm}$. The results are in good agreement with the TEM observation.

\section{FTIR analysis}

FTIR spectra analysis could help with the identification of functional groups in the samples and reveal how the composition and structure of the lettuce fibers changed during the chemical treatments. The FTIR spectra of lettuce peel bran, cellulose and CNFs are shown in Figure 4.

In the region of $3650-3000 \mathrm{~cm}^{-1}$, the broad band of stretching vibrations of $\mathrm{C}-\mathrm{H}$ and $\mathrm{O}-\mathrm{H}$ groups was observed in all the spectra, which show the principal functional groups found in cellulose materials. ${ }^{34-36}$ The peak at $1635 \mathrm{~cm}^{-1}$ corresponds to the $\mathrm{O}-\mathrm{H}$ bending of the adsorbed water. ${ }^{37}$ The shoulder at $1739 \mathrm{~cm}^{-1}$ existing in the 
lettuce peel bran was attributed to vibrations of acetyl and uronic ester groups of hemicellulose or ester linkage of carboxylic group of the ferulic acid and p-coumaric acid of lignin and/or hemicelluloses. ${ }^{38}$ This peak did not appear in the spectra of the cellulose and CNFs, since most of the hemicelluloses and lignin were removed from the samples through the bleaching procedure.

According to the cellulose spectrum, characteristic peaks of $\mathrm{C}-\mathrm{O}$ stretching vibration of alcohol hydroxyl, C-O stretching vibration of ether, skeleton vibration of ether C-C at $1060 \mathrm{~cm}^{-1}$, $1112 \mathrm{~cm}^{-1}$ and $1162 \mathrm{~cm}^{-1}$, respectively, and the peak at $898 \mathrm{~cm}^{-1}$ with increased sharpness was associated with cellulose $\beta$-glycosidic linkage. ${ }^{39}$ The peak at $1100 \mathrm{~cm}^{-1}$ was attributed to the presence of sulfuric acid groups, which was caused by sample processing. Comparing the spectra of the extracted lettuce peel cellulose and CNFs revealed that their characteristic peaks did not have significant changes, no new functional groups were generated. In the preparation process, the chemical structure of CNFs was not disrupted and changed, but still maintained the basic chemical structure of cellulose molecules.

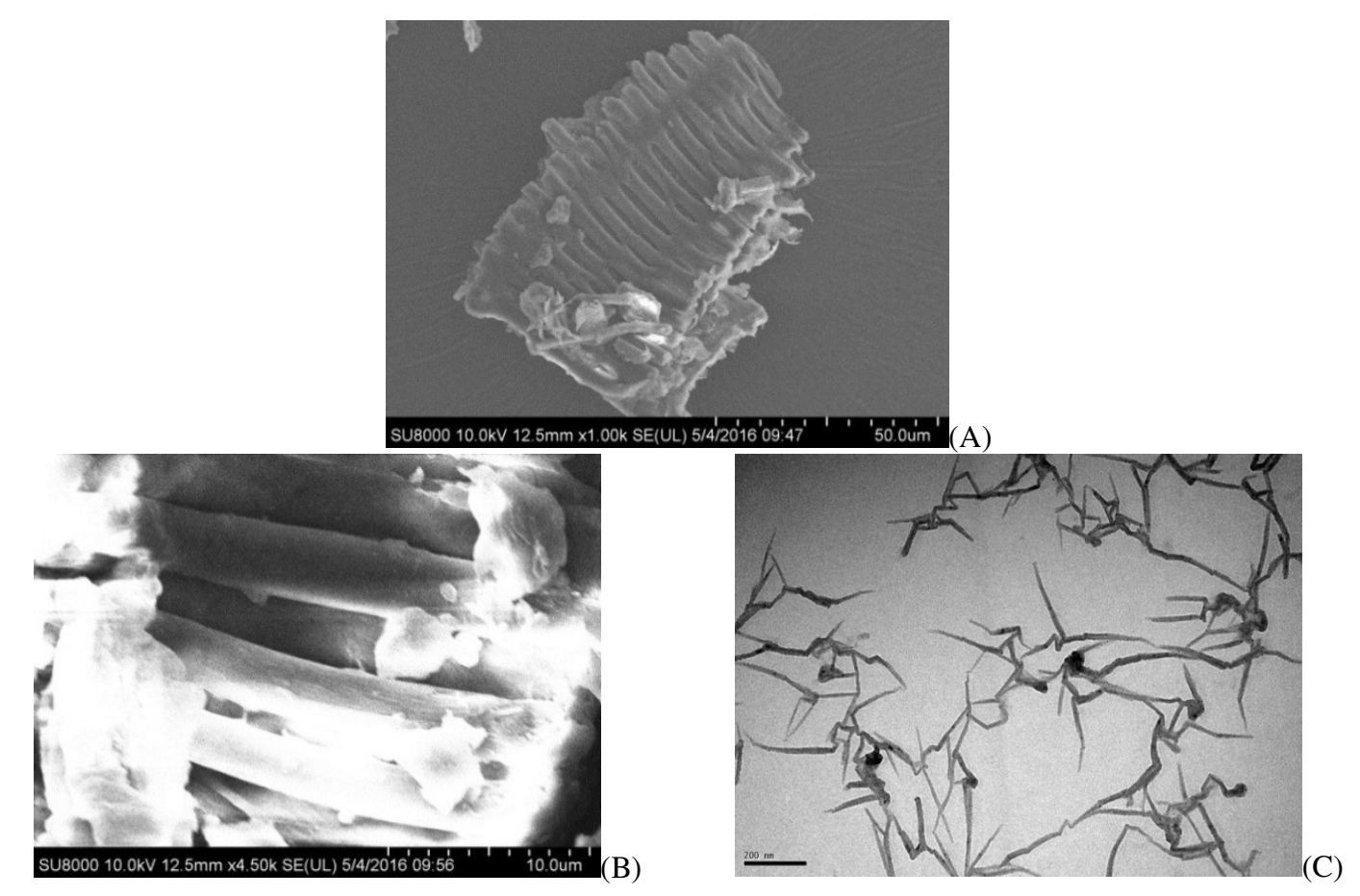

Figure 2: SEM images of lettuce peel bran (A) and cellulose extracted from lettuce peel (B); TEM image of CNFs hydrolyzed from lettuce peel cellulose (C)

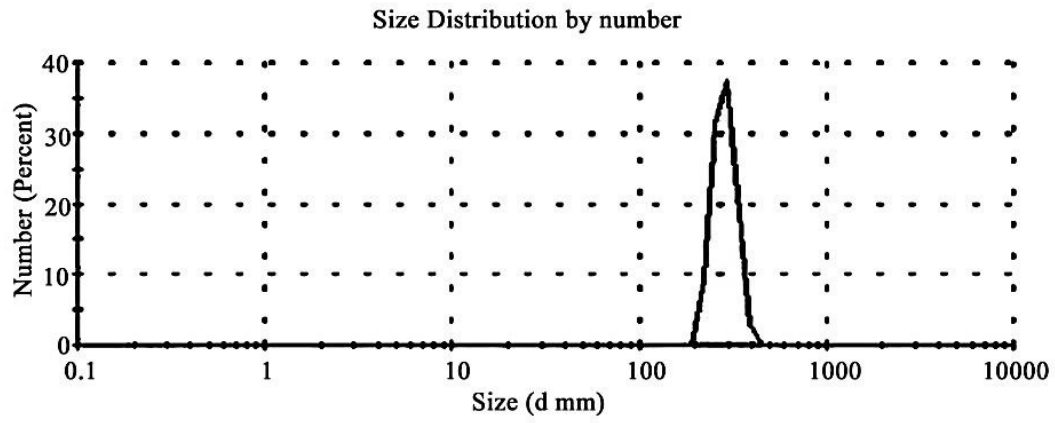

Figure 3: Particle size distribution of lettuce peel CNFs 


\section{X-ray diffraction (XRD) analysis}

The crystalline nature of different kinds of fibers obtained from lettuce peel with various treatments was investigated by the XRD method. Figure 5 shows the XRD patterns of the lettuce peel bran, lettuce peel cellulose, and CNFs prepared in this study. It can be observed in Figure 5 that all the samples exhibited a sharp diffraction peak at $2 \theta=22^{\circ}$, which is the characteristic of a typical cellulose I structure, ${ }^{15}$ which is in agreement with previous results. Chen had a similar finding using another way to isolate CNFs from tomato peel wastes. ${ }^{32}$

The intensity of the main diffraction peak at $22^{\circ}$ was stronger, which shows that the degree of crystallization was better. This is due to the alkali solution and bleaching processing, which had a large number of the hemicellulose and lignin of the non-crystalline region removed, and increased the crystallinity of cellulose from lettuce peel. During the sulfuric acid hydrolysis process, hydrogen ions entered the cellulose amorphous

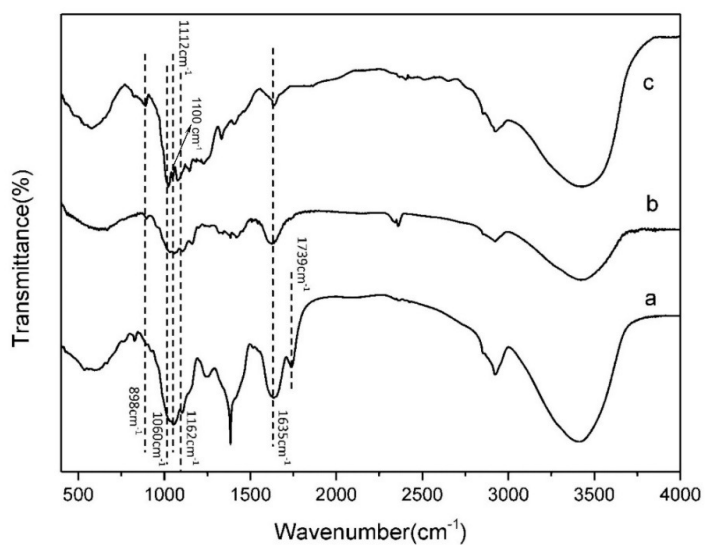

Figure 4: FTIR spectra of lettuce peel (a), lettuce peel cellulose (b), lettuce peel CNFs (c)

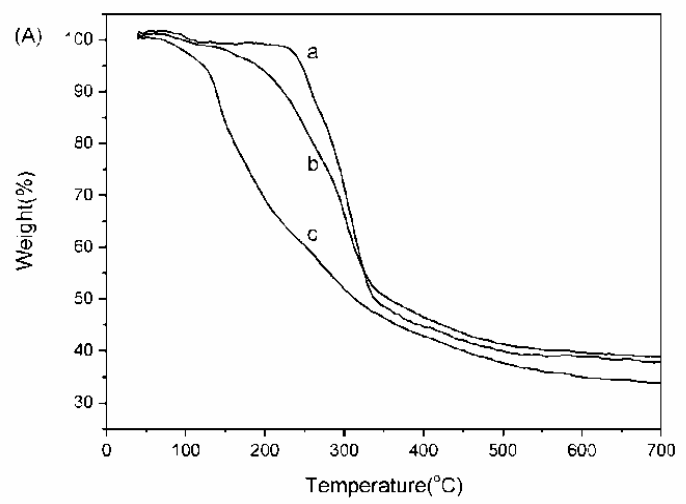

region, breaking hydrogen bonds. The amorphous region was hydrolyzed and broke into glucose etc., which belongs to water soluble substances.

At the same time, as crystalline structures were hydrolyzed, the degree of crystallinity of the lettuce peel cellulose nanofiber could be improved effectively. The crystallinity indexes (CrI) of lettuce peel, cellulose and CNFs were $19.13 \%, 31.52 \%$ and $41.57 \%$, respectively.

\section{Thermal stability}

The thermal gravimetric analysis curves of lettuce peel bran, cellulose and CNFs are presented in Figure 6. It is clear to see that all the TG curves show an initial small drop between 50 ${ }^{\circ} \mathrm{C}$ and $100{ }^{\circ} \mathrm{C}$ in Figure 6A, which corresponds to the evaporation of water molecules contained in the samples. ${ }^{40,41}$ For lettuce peel bran, the initial thermal decomposition occurred at $180^{\circ} \mathrm{C}$, followed by a sudden reduction between $180{ }^{\circ} \mathrm{C}$ and $350{ }^{\circ} \mathrm{C}$.

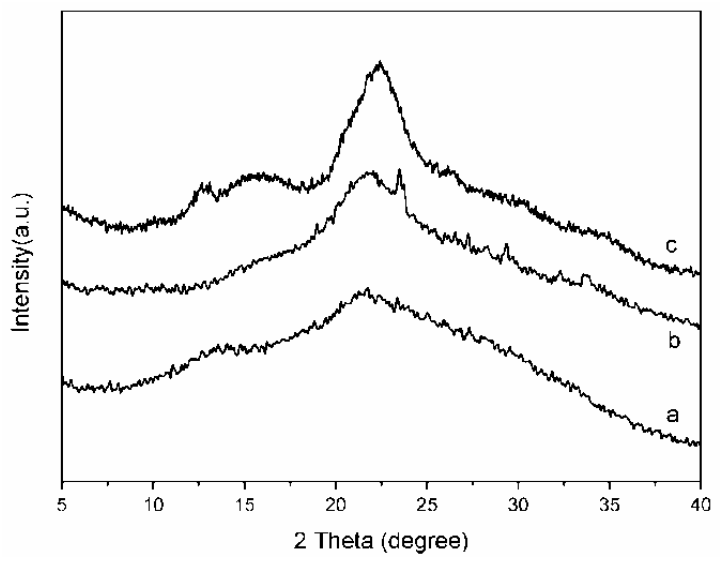

Figure 5: X-ray diffraction patterns of lettuce peel (a), lettuce peel cellulose (b), and lettuce peel CNFs (c)

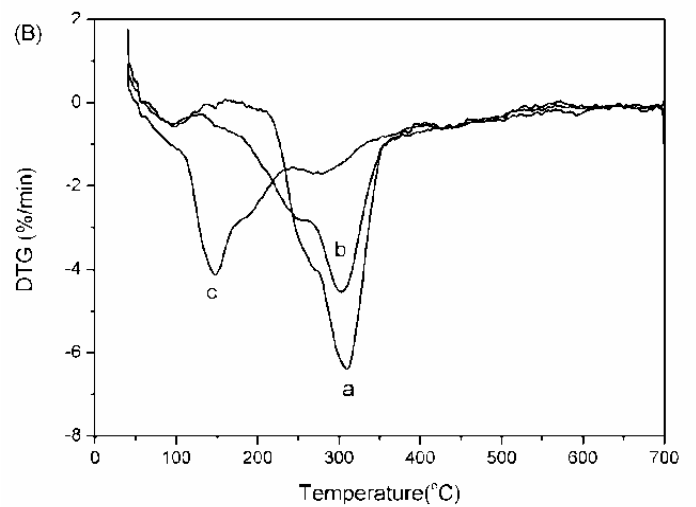

Figure 6: Thermal gravimetric analysis (TGA, A) and derivative thermogravimetric analysis (DTG, B) curves for lettuce peel (a), lettuce peel cellulose (b) and lettuce peel cellulose nanofibers (c) 
In contrast, the initial weight loss for CNFs in Figure 6A starts at $100{ }^{\circ} \mathrm{C}$ due to the presence of sulfuric acid groups on the CNF surface. These sulfuric acid groups led to dehydration reactions and decreased the activation energy of CNF chain degradation. A significant weight loss occurred in the range of $150-320{ }^{\circ} \mathrm{C}$, followed by a slow weight loss. However, for cellulose, the initial weight loss started at $250{ }^{\circ} \mathrm{C}$, generating thermal depolymerization and the cleavage of the glycosidic linkages of cellulose. ${ }^{42}$

Moreover, the derivative thermogravimetric (DTG) curves of lettuce peel bran, cellulose pulp, CNFs in Figure 6B show a major decomposition peak at $305{ }^{\circ} \mathrm{C}, 313{ }^{\circ} \mathrm{C}$ and $150{ }^{\circ} \mathrm{C}$, respectively. Comparing the three weight loss temperatures, the thermal decomposition of CNFs was greatly reduced, which might be due to the reduction of particle size and growth of specific surface areas. At the same time, surface hydroxyl groups were increased, which made the reactivity increase and led to a decrease in thermal stability. Similarly, an initial degradation temperature of CNFs was found to be of about $120{ }^{\circ} \mathrm{C} .{ }^{43}$ Furthermore, free sulfate ended on the surface of $\mathrm{H}_{2} \mathrm{SO}_{4}$ dialyzed $\mathrm{CNFs}$, which led to the lower decomposition temperature of CNFs than those of bran and cellulose. ${ }^{44}$ Also, the treatments with $\mathrm{NaOH}$, $\mathrm{NaClO}_{2}$ and sulfuric acid contributed to the decomposition or chain degradation of CNFs, which explains why the thermal stability of CNFs was lower than that of the others.

A higher amount of residue in the lettuce peel (38.8\%) was directly correlated with the presence of lignin, hemicelluloses, and extractive noncellulosic materials, which is consistent with prior reports on CNFs from other sources, such as raw bamboo, the amount of residue was $23 \% .^{13}$ From the TGA curve of cellulose in Figure 6A, only $2.23 \%$ mass loss occurred in the range of $40-180$ ${ }^{\circ} \mathrm{C}$, and a significant mass loss around $53.21 \%$ in the range of $250-380{ }^{\circ} \mathrm{C}$, which were the typical degradation characteristics of cellulose, the total mass loss was $62.3 \%$. The final residue of CNFs was lower (c) than those of lettuce peel (a) and lettuce peel cellulose (b), in addition to the removal of lignin, hemicellulose and extractive non-cellulosic materials. CNFs have a greater number of free end chains, due to their smaller particle size. Thus, the end chains start decomposition at a much lower temperature. Furthermore, it has a greater surface area compared to the other two materials, thus the final residue was lower than in the other two materials.

\section{CONCLUSION}

In this study, CNFs were obtained from lettuce peel wastes through three steps, including alkali treatment, bleaching processing and acid hydrolysis. The crystallinity index of lettuce peel cellulose rose from $19.13 \%$ to $31.52 \%$, which was characteristic of cellulose type I structure. The shape of cellulose nanofibers was needle-like, which was measured by TEM, the diameters ranged from 10 to $20 \mathrm{~nm}$ and the length varied between 190-460 nm. The initial thermal decomposition temperature was $150{ }^{\circ} \mathrm{C}$, when the temperature reached $700{ }^{\circ} \mathrm{C}$, the residual content of the lettuce peel cellulose nanofiber was greater than $30 \%$. The new isolated cellulose nanofibers are expected to be applied in the food industry or in other areas where biodegradable/biocompatible materials are sought, such as in functional materials, medical implants, etc.

ACKNOWLEDGMENTS: The authors are grateful to the financial support provided by the National Natural Science Foundation of China (31772047), the Fundamental Research Funds for the Central Universities (2014QC015) and the Scientific Research Foundation for New Recruitment of Huazhong Agricultural University (No. 52902).

\section{REFERENCES}

1 Y. Habibi, L. A. Lucia and O. J. Rojas, Chem. Rev., 110, 3479 (2010), https://doi.org/10.1021/cr900339w

2 F. Jiang, S. Han and Y. L. Hsieh, RSC Adv., 3, 12366 (2013), https://doi.org/10.1039/c3ra41646a

3 E. Abu-Danso, V. Srivastava, M. Sillanpää and A. Bhatnagar, Int. J. Biol. Macromol., 102, 248 (2017), https://doi.org/10.1016/j.ijbiomac.2017.03.172

4 B. Soni, E. B. Hassan and B. Mahmoud, Carbohyd. Polym., $\quad$ 134, $581 \quad$ (2015), https://doi.org/10.1016/j.carbpol.2015.08.031

5 S. Thambiraj and D. R. Shankaran, Appl. Surface Sci., 412, 405 (2017), http://dx.doi.org/10.1016/j.apsusc.2017.03.272

6 N. Saba, A. Safwan, M. L. Sanyang, F. Mohammad, M. Pervaiz et al., Int. J. Biol. Macromol., 102, 822

http://dx.doi.org/10.1016/j.ijbiomac.2017.04.074

7 R. Rampazzo, D. Alkan, S. Gazzotti, M. A. Ortenzi, G. Piva et al., Packag. Technol. Sci., 30, 645 (2017), https://doi.org/10.1002/pts.2308

${ }^{8}$ S. J. Eichhorn, Soft Matter, 7, 303 (2010), https://doi.org/10.1039/c0sm00142b

9 J. Feng and Y. L. Hsieh, Carbohyd. Polym., 122, 60 (2015), http://dx.doi.org/10.1016/j.carbpol.2014.12.064

${ }_{10}$ Z. H. Wang, Z. J. Yao, J. Zhou and Z. Yong, 
Carbohyd. Polym., 157, $945 \quad$ (2017), https://doi.org/10.1016/j.carbpol.2016.10.044

11 L. V. Hai and S. Yung, Cellulose Chem. Technol., 52, 741 (2018),

http://www.cellulosechemtechnol.ro/pdf/CCT910(2018)/p.741-747.pdf

12 A. D. Campos, A. C. Correa, D. Cannella, E. D. M. Teixeira, J. M. Marconcini et al., Cellulose, 20, 1491 (2013), https://doi.org/10.1007/s10570-013-9909-3

13 H. P. S. A. Khalil, M. S. Hossain, N. N. A. Nik, I. S. M. Zaidul, E. Rosamah et al., Bioresources, 9, 7710 (2014), https://doi.org/10.15376/biores.9.4.7710-7720

${ }_{14}$ S. M. L. Rosa, N. Rehman, M. I. G. D. Miranda, S. M. B. Nachtigall, C. I. D. Bica, Carbohyd. Polym., 87, 1131 (2012),

https://doi.org/10.1016/j.carbpol.2011.08.084

15 B. M. Cherian, A. L. Leão, S. F. D. Souza, S. Thomas, L. A. Pothan et al., Carbohyd. Polym., 81, 720

(2010),

https://doi.org/10.1016/j.carbpol.2010.03.046

16 P. Lu and Y. L. Hsieh, Carbohyd. Polym., 87, 2546 (2012), https://doi.org/10.1016/j.carbpol.2011.08.022

17 D. Chen, D. Lawton, M. R. Thompson and Q. Liu, Carbohyd. Polym., 90, $709 \quad$ (2012), http://dx.doi.org/10.1016/j.carbpol.2012.06.002

18 H. Tibolla, F. M. Pelissari, M. I. Rodrigues and F. C. Menegalli, Ind. Crop. Prod., 95, 664 (2017), http://dx.doi.org/10.1016/j.indcrop.2016.11.035

19 A. L. M. P. Leite, C. D. Zanon and F. C. Menegalli, Carbohyd. Polym., 157, $962 \quad$ (2017), https://doi.org/10.1016/j.carbpol.2016.10.048

20 S. Ventura-Cruz and A. Tecante, Carbohyd. Polym., 220, 53

(2019),

https://doi.org/10.1016/j.carbpol.2019.05.053

${ }^{21}$ Z. Chen, C. Zhu, Y. Zhang, D. Niu and J. Du, Postharvest Biol. Technol., 58, 232 (2010), https://doi.org/10.1016/j.postharvbio.2010.06.004

22 J. H. Lee, R. Durand, V. Gradinaru, F. Zhang, I. Goshen et al., Nature, 465, 788 (2010), https://doi.org/10.1038/nature09108

23 C. Q. Zhou and D. Yang, Journal of Southwest Agricultural University, 19, $176 \quad$ (1997), https://doi.org/10.13718/j.cnki.xdzk.1997.02.019

24 C. Yang, Y. F. Cai, R. M. Gong, D. H. Shao and Y. Z. Sun, Journal of Nanjing Agricultural University, 29, 45 (2006), https://doi.org/10.3321/j.issn:10002030.2006.02.010

25 C.-L. Du and Y.-Q. Hu, Storage Process., 6, 42 (2007), https://doi.org/10.3969/j.issn.10096221.2007.06.013

26 E. Dinand, H. Chanzy and M. R. Vignon, Cellulose, 3, 183 (1996), https://doi.org/10.1007/bf02228800

27 M. F. Rosa, E. S. Medeiros, J. A. Malmonge, K. S. Gregorski, D. F. Wood et al., Carbohyd. Polym., 81, 83 (2010), https://doi.org/10.1016/j.carbpol.2010.01.059

${ }_{28}$ H. Tibolla, F. M. Pelissari and F. C. Menegalli, LWT-Food Sci. Technol., 59, 1311 (2014), https://doi.org/10.1016/j.lwt.2014.04.011

29 B. Soni, E. B. Hassan and B. Mahmoud, Carbohyd.
Polym., $\quad$ 134, $581 \quad$ (2015), 10.1016/j.carbpol.2015.08.031

30 L. C. Segal, J. Creely, A. E. J. Martin and C. M. Conrad, Textile Res. J., 29, 786 (1959), https://doi.org/10.1177/004051755902901003

31 M. Li, L. J. Wang, D. Li, Y. L. Cheng and B. Adhikari, Carbohyd. Polym., 102, 136 (2014), http://dx.doi.org/10.1016/j.carbpol.2013.11.021

32 P. Khawas and S. C. Deka, Carbohyd. Polym., 137, 608

(2016),

http://dx.doi.org/10.1016/j.carbpol.2015.11.020

33 W. Chen, H. Yu, Y. Liu, P. Chen, M. Zhang et al., Carbohyd. Polym., 83, $1804 \quad$ (2011), https://doi.org/10.1016/j.carbpol.2010.10.040

34 T. F. Meyabadi and F. Dadashian, Fiber. Polym., 13, 313 (2012), https://doi.org/10.1007/s12221-0120313-7

35 W. Kamphunthong, P. Hornsby and K. Sirisinha, J. Appl. Polym. Sci., 125, $1642 \quad$ (2012), https://doi.org/10.1002/app.35642

${ }_{36}$ J. Q. Han, C. J. Zhou, Y. Q. Wu, F. Y. Liu and Q. L. Wu, Biomacromolecules, 14, 1529 (2013), https://doi.org/10.1021/bm4001734

37 R. Zuluaga, J. L. Putaux, J. Cruz, J. Vélez, I. Mondragon et al., Carbohyd. Polym., 76, 51 (2009), https://doi.org/10.1016/j.carbpol.2008.09.024

38 X. F. Sun, F. Xu, R. C. Sun, P. Fowler and M. S. Baird, Carbohyd. Res., 340, 97 (2005), https://doi.org/10.1016/j.carres.2004.10.022

39 I. Siró and D. Plackett, Cellulose, 17, 459 (2010), https://doi.org/10.1007/s10570-010-9405-y

${ }^{40}$ A. Kumar, V. Choudhary, N. K. Bhardwaj and Y. S. Negi, J. Mater. Phys. Chem., 2, 1 (2014), https://doi.org/10.1007/s10570-014-0339-7

41 W. Li, R. Wang and S. Liu, Bioresources, 6, 4271 (2011),

https://bioresources.cnr.ncsu.edu/BioRes_06/BioRes_0 6_4_4271_Li_Wang_Liu_Nanocryst_Cell_SW_Kraft_ Ultrasonic_Hydrol_1896.pdf

${ }^{42}$ B. Deepa, E. Abraham, B. M. Cherian, A. Bismarck, J. J. Blaker et al., Bioresour. Technol., 102, 1988 https://doi.org/10.1016/j.biortech.2010.09.030

${ }^{43}$ K. Oksman, J. A. Etang, A. P. Mathew and M. Jonoobi, Biomass Bioenerg., 35, 146 (2011), https://doi.org/10.1016/j.biombioe.2010.08.021

44 Y. Zhang and H. Zhao, Polymer, 64, 277 (2015), https://doi.org/10.1016/j.polymer.2015.02.005 